05,13

\title{
Латеральный спин-волновой транспорт в системе неидентичных магнонно-кристаллических микроволноводов
}

\author{
(C) В.А. Губанов, С.Е. Шешукова, А.В. Садовников \\ Саратовский государственный университет им. Н.Г. Чернышевского, \\ Саратов, Россия \\ E-mail: vladmeen@gmail.com
}

Поступила в Редакцию 9 апреля 2021 г.

В окончательной редакции 9 апреля 2021 г.

Принята к публикации 19 апреля 2021 г.

\begin{abstract}
Методом мандельштам-бриллюэновской спектроскопии и методом микромагнитного моделирования исследованы режимы управления дипольной связью в латеральной системе неидентичных магнонных кристаллов. Выявлены режимы пространственной и частотной селекции спин-волнового сигнала вблизи частоты зоны непропускания магнонного кристалла. Исследовано влияние изменения геометрических параметров на свойства волноводных мод вблизи частоты брэгговского резонанса. Полученные результаты могут быть использованы для создания устройств обработки СВЧ-сигнала, таких как демультиплексеров, делителей мощности, ответвителей.
\end{abstract}

Ключевые слова: спиновые волны, магноника, латеральные структуры, магнонный кристалл.

DOI: 10.21883/FTT.2021.09.51309.31H

\section{1. Введение}

В последнее время активно развивается новое направление спинтроники с использованием ферритов-гранатов - магноника [1], в рамках которой транспортные свойства спин-поляризованных электронов не используются, а перенос информации происходит путем передачи сигнала с помощью спиновых волн (СВ) [2]. При таком подходе удается реализовать целый ряд функциональных блоков обработки сигнала на принципах магноники $[3,4]$. Малая константа затухания СВ в пленках железоиттриевого граната (YIG) толщинами от единиц микрон и вплоть до $20 \mathrm{~nm}$, выращенных методами жидко-фазной эпитаксии [5] либо техникой импульсного лазерного осаждения [6,7] позволяет существенно минимизировать потери при распространении сигнала, закодированного в виде амплитуды и фазы СВ. Диэлектрические свойства YIG позволяют существенно уменьшить омические потери по сравнению с металлическими ферромагнитными пленками.

Управление свойствами СВ возможно путем создания нерегулярных тонкопленочных структур с нарушением свойств трансляционной симметрии $[8,9]$, локально индуцированных деформаций [10] или создания периодических в направлении распространения CВ-структур магнонных кристаллов (МК). МК активно исследуются благодаря возможности формирования запрещенных зон [11]. При этом путем создания составных структур на основе тонких магнитных пленок удается управлять свойствами СВ при использовании геометрических эффектов [12]. Последние используются для подходов градиентной магноники $[13,14]$ и концепции построения трехмерных магнонных сетей $[15,16]$. Использование латерального спин-волнового транспорта в магнонных структурах находит применение для реализации режимов пространственно-частотной селекции информационных сигналов, реализующемся, например, в структуре, состоящей из неидентичных МК [17]. В последнем случае представляет интерес рассмотреть эффекты пространственно-частотного разделения спин-волнового сигнала при отдельном возбуждении каждого из МК, что и являлось целью настоящей работы.

В настоящей работе на основе численного микромагнитного моделирования и экспериментального исследования методом мандельштам-бриллюэновской спектроскопии показана возможность одновременного использования свойств дипольной спин-волновой связи и многомодового режима распространения СВ в связанной латеральной системе неидентичных магнонных кристаллов на частотах, близких к полосе непропускания МК.

\section{2. Исследуемые структуры и методика численного моделирования}

На рис. 1 показано схематическое изображение исследуемой структуры, состоящая из двух МК (MC1 и $M C 2)$, сориентированных вдоль направления оси $y$. Латеральный зазор между боковыми стенками магнонных кристаллов $M C 1$ и $M C 2 d=20 \mu \mathrm{m}$. Каждый МК представляет собой структуру с канавками глубиной $1 \mu \mathrm{m}$, повторяющимися с периодом $D=200 \mu \mathrm{m}$. Длина участков с периодической системой канавок составляет величину $L_{c}=5 \mathrm{~mm}$. Ширины $M C 1$ и $M C 2$ вдоль оси $z$ составляли $w_{1}=200 \mu \mathrm{m}$ и $w_{2}=500 \mu \mathrm{m}$, соответственно. В качестве материала для изготовления магнонных кристаллов была использована пленка железо-иттриевого граната (YIG) $\mathrm{Y}_{3} \mathrm{Fe}_{5} \mathrm{O}_{12}$ [18] толщиной $t=10 \mu \mathrm{m}$ с намагни- 


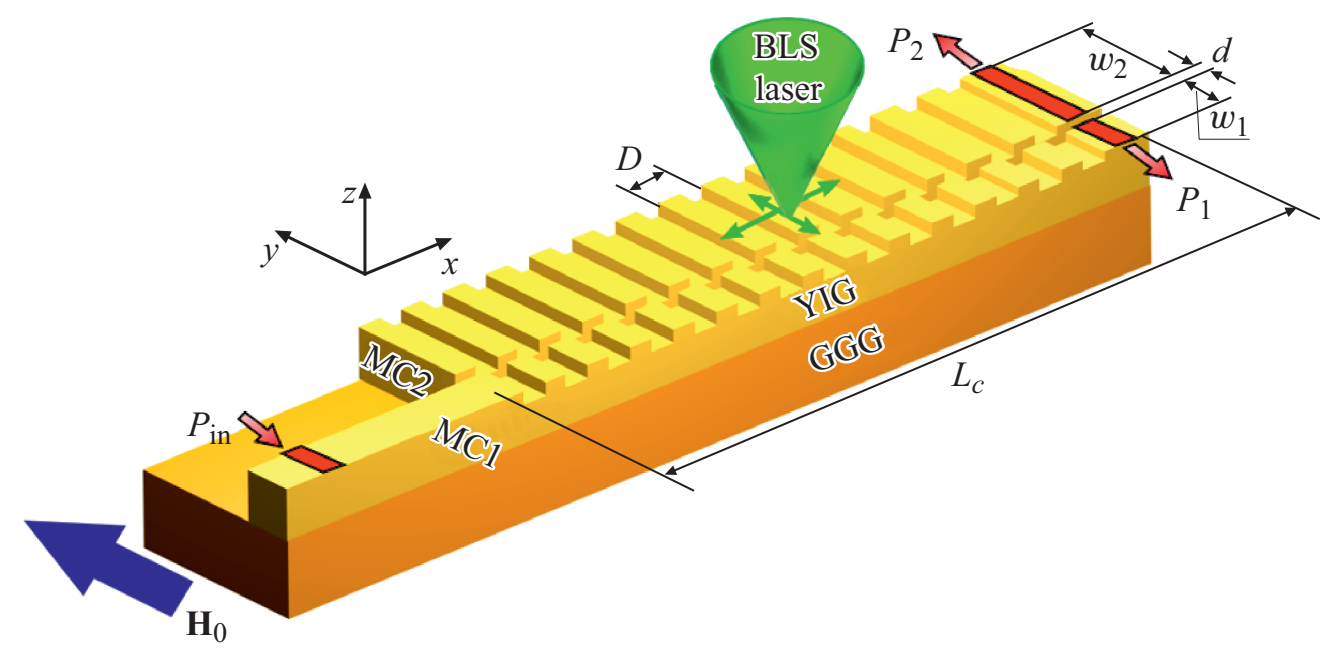

Рис. 1. Изображение исследуемой структуры

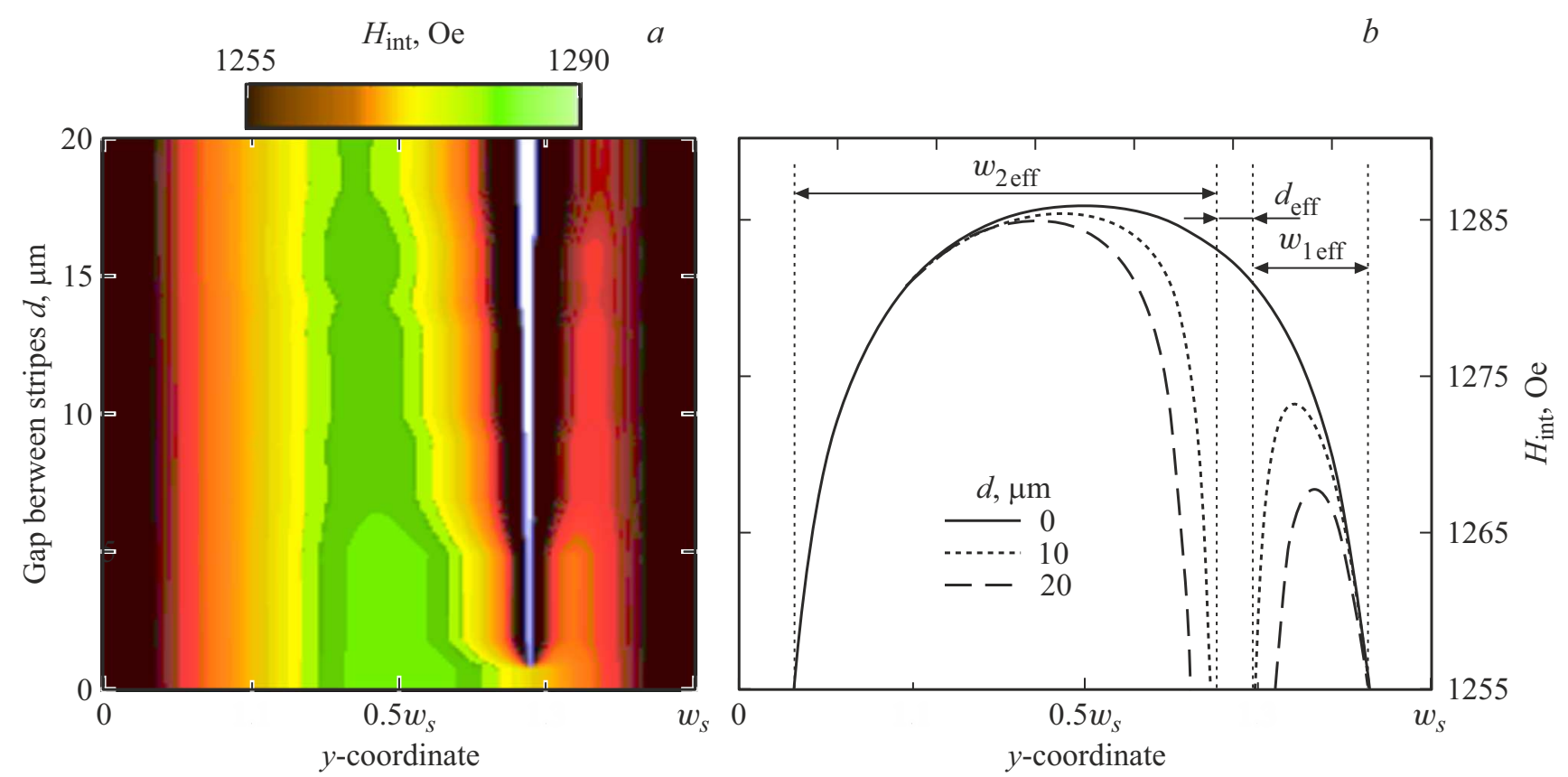

Рис. 2. Карта распределения величины внутреннего магнитного поля вдоль оси $у$ в зависимости от расстояния между магнонными кристаллами $M C 1$ и $M C 2(a)$. Профили внутреннего магнитного поля в зависимости от расстояния между магнонными кристаллами $M C 1$ и $M C 2(b)$.

ченностью насыщения $M_{0}=139 \mathrm{G}$ и шириной линии ферромагнитного резонанса $\Delta H=0.54 \mathrm{Oе}$, измеренной на частоте $9.7 \mathrm{GHz}$. YIG выращен методом высокотемпературной жидкофазной эпитаксии на подложке галлийгадолиниевого граната $(\mathrm{GGG}) \mathrm{Gd}_{3} \mathrm{Ga}_{5} \mathrm{O}_{12}$. Пленка была структурирована с помощью установки локальной лазерной абляции на основе волоконного YAG: Nd лазеpa c 2D гальванометрическим сканирующем модулем (Cambridge Technology 6240H), работающем в импульсном режиме с длительностью импульса $50 \mathrm{~ns}$ и мощностью $50 \mathrm{~mJ}$. Метод был адаптирован для использования на пленках YIG с толщинами $0.1-10 \mu \mathrm{m}$.
Структура помещалась между полюсами электромагнита GMW 3472-70, при этом ориентация магнитного поля совпадала с направлением оси $y$ (см. рис. 1). В такой конфигурации на входную антенну $P_{\text {in }}$, расположенной на $M C 1$, подавался СВЧ-сигнал с генератора Anritsu MG3692C. В данной геометрии вдоль МК возможным оказывалось возбуждение поверхностной магнитостатической волны (ПМСВ) $[19,20]$. Прием сигнала осуществлялся выходными антеннами $P_{1}$ и $P_{2}$ на $M C 1$ и $M C 2$, соответственно. Ширины микрополосковых антенн $P_{\text {in }}$, $P_{1}$ и $P_{2}$ составляли $30 \mu \mathrm{m}$. Величина прикладываемого внешнего магнитного поля $H_{0}=1300 \mathrm{Oe}$. 
Исследование стационарного пространственного распределения динамической намагниченности было проведено методом Мандельштам-Бриллюэновской спектроскопии (Brillouin light scattering (BLS)), в основе которого лежит эффект неупругого рассеяния света на когерентно возбуждаемых магнонах [21,22]. Эксперимент проводился в конфигурации квазиобратного рассеяния, интенсивность оптического сигнала при этом оказывается пропорциональной квадрату динамической намагниченности в пространственной области, где сфокусирован лазерный луч на поверхности пленки YIG: $I_{\mathrm{BLS}}=\sqrt{m_{z}^{2}+m_{x}^{2}}$.

Экспериментальные результаты сопоставлялись с результатами микромагнитного моделирования (MM), проведённые в программе MuMax3 [23] на основе численного решения уравнения Ландау-ЛифшицаГильберта $[24,25]$. Метод ММ позволяет численно решить задачу о возбуждении и распространении СВ в системе латерально связанных неидентичных магнонных кристаллов. Определяя область возбуждения и параметры входного сигнала в МК можно наблюдать стационарный режим установления волнового процесса в структуре. При расчете размер ячейки численной сетки был положен $0.2 \times 0.7 \times 10 \mu \mathrm{m}$.

Был проведен расчет пространственных зависимостей величины внутреннего магнитного поля $H_{\text {int }}(y)$ для значений величины зазора между магнонными кристаллами $0<d<20 \mu \mathrm{m}$ (рис. 2, $a, b$ ). На оси абсцисс отложено значение координаты $y$ в диапазоне $0<y<w_{s}$, где $w_{s}=w_{1}+w_{2}+d$. Из анализа профилей $H_{\text {int }}(y)$, показанных на рис. 2, $b$, следует что при увеличении зазоpa $d$ наблюдается уменьшение величины $H_{\mathrm{int}}\left(y=y_{c 1}\right)$, где координата $y_{c 1}$ соответствует центру MC1. Изменение величины внутреннего поля сопровождается изменением спектра ПМСВ в $M C 1,2$ и изменением коэффициента связи $\mathrm{CB}$, распространяющихся вдоль латеральной структуры. Последнее является следствием изменения величины эффективного зазора $d_{\text {eff }}$ и эффек-

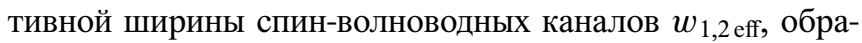
зующихся при касательном намагничивании $M C 1,2$ [26]. Эффективная ширина $w_{1,2 \text { eff }}$ меньше чем геометрическая ширина волновода за счет существования полей размагничивания [22] При этом ширина эффективного зазора $d_{\text {eff }}$ увеличивается. Ввиду этого при экспериментальном изготовлении латеральных структур можно пользоваться эмпирическим правилом для выбора величины зазора: $d \sim 2 t$, которое обеспечивает наиболее эффективную передачу энергии СВ между магнонными кристаллами [4].

Возбуждение СВ в $M C 1$ происходило на частоте $f_{\mathrm{B}}=5.6 \mathrm{GHz}$. При этом мощность входного сигнала составляла величину $P_{0}=-15 \mathrm{dBmW}$, что обеспечивало линейный режим возбуждения и распространения СВ вдоль структуры. Эксперимент осуществлялся в импульсном режиме: сигнал имел форму прямоугольных импульсов длительностью $200 \mathrm{~ns}$ с периодом повторения $2 \mu \mathrm{s}$. Пространственное сканирование осуществлялось в области $2.5 \times 0.73 \mathrm{~mm}^{2}$.
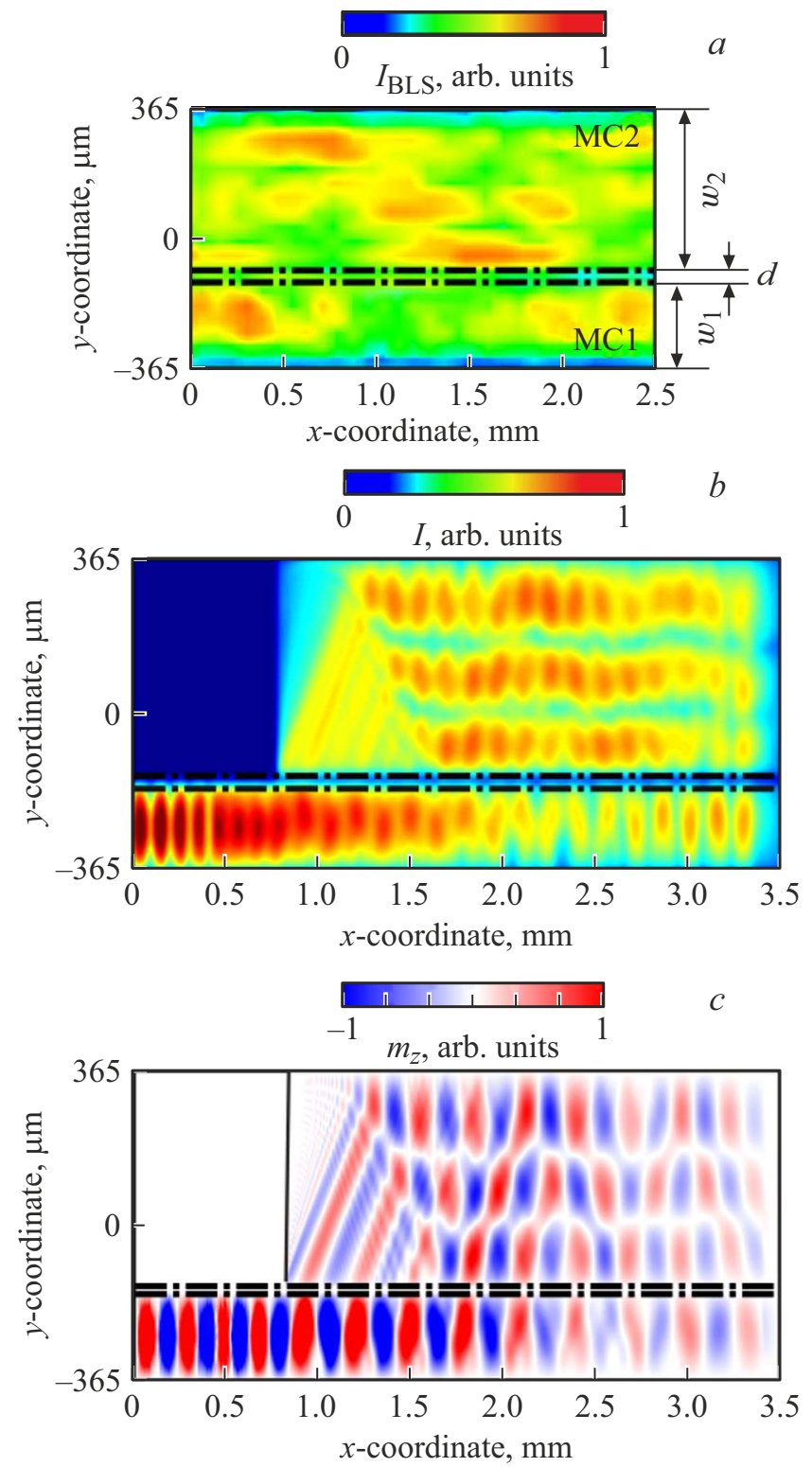

Рис. 3. Результаты BLS-эксперимента: пространственное распределение интенсивности спиновой волны $I(x, y)$ в случае возбуждения волны в магнонном кристалле $M C 1(a)$. Результаты микромагнитного моделирования: пространственное распределение интенсивности $I(x, y)$ и компоненты динамической намагниченности $m_{z}(x, y)$ в случае возбуждения СВ в магнонном кристалле $M C 1(b, c)$.

На рис. 3, а показаны пространственные карты распределения интенсивности $I_{\mathrm{BLS}}(x, y)$ для случая возбуждения $\mathrm{CB}$ в узком (MC1) MК на частоте входного

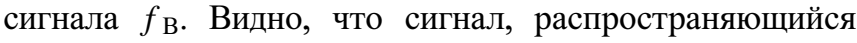
на некоторое расстояние от антенны, перекачивается в смежный МК, и распространяется вдоль него.

Полученные методом ММ карты распределения величины интенсивности спиновой волны $I(x, y)=I_{\mathrm{BLS}}$ (рис. $3, b)$ и ортогональной поверхности пленки компоненты намагниченности $m_{z}$ (рис. $\left.3, c\right)$ находятся в хоро- 


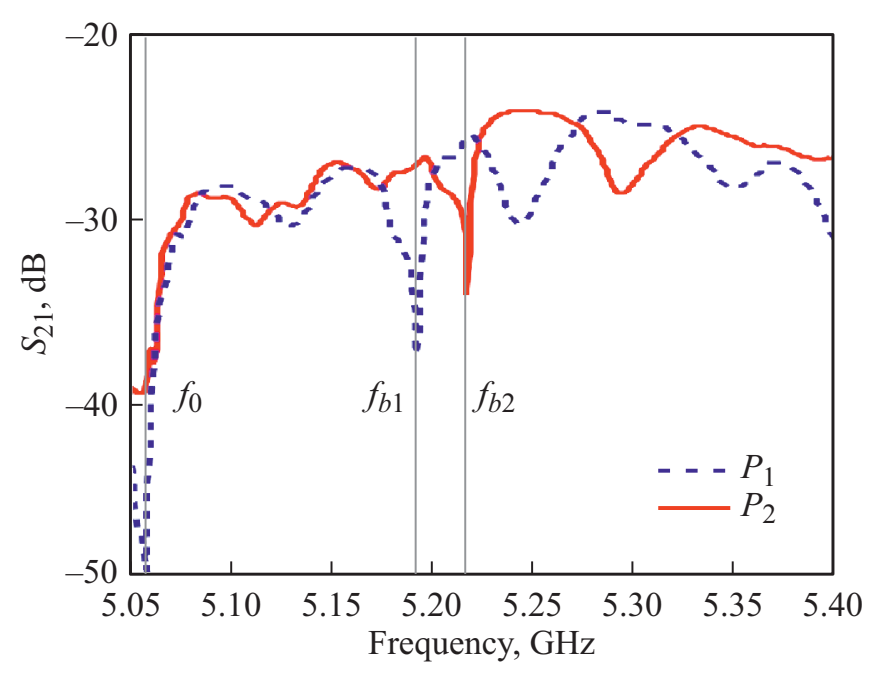

Рис. 4. Полученные методом микромагнитного моделирования АЧХ, снятые с портов $P_{1}$ и $P_{2} M C 1$ и $M C 2$ соответственно.

шем соответствии с данными, полученными в эксперименте. Так, проводя модовую декомпозицию карты пространственного распределения интенсивности [19] СВ на рис. 3 можно отметить, что в возбуждаемом волноводе наблюдается преимущественно распространение сигнала с поперечным профилем, близким к первой ширинной моде, в то время как в диапазоне $0.75<x<2 \mathrm{~mm}$, когда в $M C 1$ сигнал отсутствует, в $M C 2$ можно выделить режим распространения третьей ширинной моды.

Для определения режимов работы латеральных МК в качестве пространственно-частотного разделителя спинволновой мощности и направленного ответвителя спинволнового сигнала был проведен расчет передаточных характеристик в областях, соответствующих каждой из выходных антенн $\left(P_{1}\right.$ и $\left.P_{2}\right)$. На рис. 4 представлены частотные зависимости спектральной плотности мощности спин-волнового сигнала $S_{21}(f)$ в исследуемой структуре связанных неидентичных МК. Расчет выполнен методом ММ при возбуждении $M C 1$ импульсным сигналом и проведением преобразования Фурье. На рис. 4 синей пунктирной и красной сплошной кривыми отмечены частотные зависимости $S_{21}(f)$ для областей выходных антенн $P_{1}$ и $P_{2}$ структур $M C 1$ и $M C 2$ соответственно. Видно, что частота начала спектра СВ для обоих МК совпадает и составляет $f_{0}=\gamma\left(H_{\text {int }}\left(H_{\text {int }}+4 \pi M_{0}\right)\right)^{1 / 2} \approx 5.051 \mathrm{GHz}$. Важно отметить, что частоты провалов $f_{b 1}$ и $f_{b 2}$ в спектрах прохождения, характерных для запрещенных периодических структур $M C 1$ и $M C 2$, смещены относительно друг друга на величину $30 \mathrm{MHz}$, что может быть использовано для пространственно-частотной селекции сигнала, подаваемого на вход $P_{\text {in }}$ структуры.

\section{3. Заключение}

Таким образом, с помощью метода мандельштамбриллюэновской спектроскопии и численного моделиро- вания исследованы режимы распространения связанных СВ в системе латерально связанных неидентичных магнонных кристаллов. Продемонстрирована возможность управления межмодовой связью волн, распространяющихся в магнонных кристаллах вблизи границы частоты зоны непропускания. На основе результатов микромагнитного моделирования определен механизм, сопровождающий наблюдаемый режим межмодовой связи в латеральной системе неидентичных МК, и проведено исследование режимов пространственно-частотного разделения сигнала. Полученные результаты демонстрируют возможность создания демультиплексора и направленного ответвителя на основе латеральной системы неидентичных магнонных кристаллов.

\section{Финансирование работы}

Работа выполнена при поддержке Минобрнауки России в рамках выполнения государственного задания (проект № FSRR-2020-0005).

\section{Конфликт интересов}

Авторы заявляют, что у них нет конфликта интересов.

\section{Список литературы}

[1] С.А. Никитов, Д.В. Калябин, И.В. Лисенков, А.Н. Славин, Ю.Н. Барабаненков, С.А. Осокин, А.В. Садовников, Е.Н. Бегинин, М.А. Морозова, Ю.П. Шараевский, Ю.А. Филимонов, Ю.В. Хивинцев, С.Л. Высоцкий, В.К. Сахаров, Е.С. Павлов. УФН 185, 1099 (2015). [S.A. Nikitov, D.V. Kalyabin, I.V. Lisenkov, A.N. Slavin, Y.N. Barabanenkov, S.A. Osokin, A.V. Sadovnikov, E.N. Beginin, M.A. Morozova, Y.P. Sharaevsky, Y.A. Filimonov, Y.V. Khivintsev, S.L. Vysotsky, V.K. Sakharov, E.S. Pavlov. Phys. Usp. 58, 1002 (2015)].

[2] V. Demidov, S. Urazhdin, G. de Loubens, O. Klein, V. Cros, A. Anane, S. Demokritov. Phys. Rep. 673, 1 (2017).

[3] C.S. Davies, A.V. Sadovnikov, S.V. Grishin, Y.P. Sharaevsky, S.A. Nikitov, V.V. Kruglyak. IEEE Trans. Magn. 51, 1 (2015).

[4] А.В. Садовников, А.А. Грачев, С.А. Одинцов, А.А. Мартышкин, В.А. Губанов, С.Е. Шешукова, С.А. Никитов. Письма в ЖЭТФ 108, 5, 332 (2018). [A.V. Sadovnikov, A.A. Grachev, S.A. Odintsov, A.A. Martyshkin, V.A. Gubanov, S.E. Sheshukova, S.A. Nikitov. JETP Lett. 108, 312 (2018)].

[5] C. Dubs, O. Surzhenko, R. Thomas, J. Osten, T. Schneider, K. Lenz, J. Grenzer, R. Hübner, E. Wendler. Phys. Rev. Mater. 4, 024416 (2020).

[6] Y. Sun, Y.-Y. Song, H. Chang, M. Kabatek, M. Jantz, W. Schneider, M. Wu, H. Schultheiss, A. Hoffmann. Appl. Phys. Lett. 101, 152405 (2012).

[7] L.V. Lutsev, A.M. Korovin, S.M. Suturin, L.S. Vlasenko, M.P. Volkov, N.S. Sokolov. J. Phys. D 53, 265003 (2020).

[8] В.А. Губанов, А.А. Мартышкин, С.Е. Шешукова, А.В. Садовников. ЖТФ 89, 11, 1726 (2019). [V. A. Gubanov, A.A. Martyshkin, S.E. Sheshukova, A.V. Sadovnikov. Tech. Phys. 64, 1636 (2019)]. 
[9] A.V. Sadovnikov, C.S. Davies, V.V. Kruglyak, D.V. Romanenko, S.V. Grishin, E.N. Beginin, Y.P. Sharaevskii, S.A. Nikitov. Phys. Rev. B 96, 060401(R) (2017).

[10] V. Sadovnikov, A.A. Grachev, S.E. Sheshukova, Y.P. Sharaevskii, A.A. Serdobintsev, D.M. Mitin, S.A. Nikitov. Phys. Rev. Lett. 120, 257203 (2018).

[11] S. Nikitov, P. Tailhades, C. Tsai. J. Magn. Magn. Mater. 236, 320 (2001).

[12] I.A. Golovchanskiy, N.N. Abramov, V.S. Stolyarov, V.V. Ryazanov, A.A. Golubov, A.V. Ustinov. J. Appl. Phys. 124, 233903 (2018).

[13] A.V. Sadovnikov, C.S. Davies, V.V. Kruglyak, D.V. Romanenko, S.V. Grishin, E.N. Beginin, Y.P. Sharaevskii, S.A. Nikitov. Phys. Rev. B. 96, 060401 (2017).

[14] S.L. Vysotskii, A.V. Sadovnikov, G.M. Dudko, A.V. Kozhevnikov, Y.V. Khivintsev, V.K. Sakharov, N.N. Novitskii, A.I. Stognij, Y.A. Filimonov. Appl. Phys. Lett. 117, 102403 (2020)

[15] E. Beginin, D. Kalyabin, P. Popov, A. Sadovnikov, A. Sharaevskaya, A. Stognij, S. Nikitov. In: Three-Dimensional Magnonics / Ed. G. Gubbiotti. Jenny Stanford Publishing, Singapore (2019). P. 67.

[16] V.K. Sakharov, E.N. Beginin, Y.V. Khivintsev, A.V. Sadovnikov, A.I. Stognij, Y.A. Filimonov, S.A. Nikitov. Appl. Phys. Lett. 117, 022403 (2020).

[17] A.V. Sadovnikov, V.A. Gubanov, S.E. Sheshukova, Y.P. Sharaevskii, S.A. Nikitov. Phys. Rev. Appl. 9, 051002 (2018).

[18] V. Cherepanov, I. Kolokolov, V. Lvov. Phys. Rep. 229, 81 (1993).

[19] R. Damon, J. Eshbach. J. Phys. Chem. Solids 19, 308 (1961).

[20] S.N. Bajpai. J. Appl. Phys. 58, 910 (1985).

[21] V.E. Demidov, O. Dzyapko, S.O. Demokritov, G.A. Melkov, A.N. Slavin. Phys. Rev. Lett. 100, 047205 (2008).

[22] S. Demokritov. Phys. Rep. 348, 441 (2001).

[23] A. Vansteenkiste, J. Leliaert, M. Dvornik, M. Helsen, F. Garcia-Sanchez, B.V. Waeyenberge. AIP Advances 4, 107133 (2014).

[24] L. Landau, E. Lifshitz. Phys. Z. Sow. 8, 153 (1935).

[25] T.L. Gilbert, J.M. Kelly. In: Conf. Magnetism and Magnetic Materials. Pittsburgh, PA. American Institute of Electrical Engineers. N.Y. (1955). P. 253-263.

[26] K.Y. Guslienko, S.O. Demokritov, B. Hillebrands, A.N. Slavin. Phys. Rev. B 66, 132402 (2002).

Редактор Ю.Э. Китаев 